\title{
Cost-Effectiveness of Rotavirus Vaccination for Under-Five Children in
} Iran

\author{
Sareh Shakerian ${ }^{1}$; Maziar Moradi Lakeh ${ }^{2}$; Abdoulreza Esteghamati ${ }^{3}$; Mohsen Zahraei ${ }^{4}$; \\ Mohsen Yaghoubi ${ }^{5,}$ \\ ${ }^{1}$ School of Medical Education, Shahid Beheshti University of Medical Sciences and Health Services, Tehran, IR Iran \\ ${ }^{2}$ Department of Community Medicine, Gastro-Intestinal and Liver Disease Research Center, Iran University of Medical Sciences, Tehran, IR Iran \\ ${ }^{3}$ Department of Pediatrics, Akbarabadi Hospital, Iran University of Medical Sciences, Tehran, IR Iran \\ ${ }_{5}^{4}$ Ministry of Health and Medical Education, Tehran, IR Iran \\ ${ }^{5}$ Clinical Knowledge Management Unit, Department of Community Medicine, School of Medicine, Iran University of Medical Sciences, Tehran, IR Iran \\ ${ }^{*}$ Corresponding author: Mohsen Yaghoubi, Clinical Knowledge Management Unit, Department of Community Medicine, School of Medicine, Iran University of Medical Sciences, \\ Tehran, IR Iran. Tel: +98-2188602225, Fax: +98-2188602225, E-mail: mohsenyaq@yahoo.com \\ Received: June 7, 2015; Accepted: June 21, 2015
}

\begin{abstract}
Background: Rotavirus diarrhea is one of the most important causes of death among under-five children. Anti-rotavirus vaccination of these children may have a reducing effect on the disease.

Objectives: this study is intended to contribute to health policy-makers of the country about the optimal decision and policy development in this area, by performing cost-effectiveness and cost-utility analysis on anti-rotavirus vaccination for under-5 children.

Patients and Methods: A cost-effectiveness analysis was performed using a decision tree model to analyze rotavirus vaccination, which was compared with no vaccination with Iran's ministry of health perspective in a 5-year time horizon. Epidemiological data were collected from published and unpublished sources. Four different assumptions were considered to the extent of the disease episode. To analyze costs, the costs of implementing the vaccination program were calculated with $98 \%$ coverage and the cost of USD 7 per dose. Medical and social costs of the disease were evaluated by sampling patients with rotavirus diarrhea, and sensitivity analysis was also performed for different episode rates and vaccine price per dose.

Results: For the most optimistic assumption for the episode of illness (10.2 per year), the cost per DALY averted is 12,760 and 7,404 for RotaTeq and Rotarix vaccines, respectively, while assuming the episode of illness is 300\%, they will be equal to 2,395 and 354, respectively, which will be highly cost-effective. Number of life-years gained is equal to 3,533 years.

Conclusions: Assuming that the illness episodes are $100 \%$ and 300\% for Rotarix and 300\% for Rota Teq, the ratio of cost per DALY averted is highly cost-effective, based on the threshold of the world health organization ( $<1 \mathrm{GDP}$ per capita $=4526 \mathrm{USD})$. The implementation of a national rotavirus vaccination program is suggested.
\end{abstract}

Keywords: Rotavirus; Acute Diarrhea; Cost-Effectiveness; Iran; Children

\section{Background}

Acute diarrhea is responsible for nearly 1.9 million deaths per year in under-5 children (1). Disease caused by the virus varies from very mild disease with watery diarrhea to severe diarrhea with the loss of body water and electrolytes along with shock and death. During the first 5 years of life, each person may be infected with the virus at least once, which would result in one out of every five cases of referring to the clinic, one out of every 65 cases of hospitalization be responsible for approximately forty percent of all cases of severe diarrhea in infants (2-6). The virus causes $527,000(475,000-580,000)$ deaths per year in under-2 children, almost all of which occur in developing countries (3-7). The latest statistics of the virus in Iran indicates that rotavirus in diarrheal cases is positive in more than $59.1 \%$ of hospital visits (7). Global incidence of rotavirus infections showed that the provision of clean water and good sanitation are less able to be effective in reducing the virus trans- mission, and vaccination is the only effective primary prevention. It is important to note that over $85 \%$ of deaths from the disease occur in developing countries in Africa and Asia (6-12). High vaccine prices, along with the lack of awareness of the burden of disease and the efficacy and safety of the vaccine, has resulted in less use of the vaccine in routine vaccination programs of the low- and medium-income countries $(9,13,14)$. Developing and low-income countries are facing financial challenges in the areas of purchasing and keeping new vaccines (12, 13, 15-17). In Iran, many studies have shown the relatively high incidence of the disease, especially in diarrhea hospitalizations, which causes a high burden of disease and the occupancy of hospital beds. In addition to above cases, the limited budgets of the ministry of health also require the need for studies on the costeffectiveness, in order to prioritize and strengthen policies for the implementation of prevention programs.

Copyright (C) 2015, Growth \& Development Research Center. This is an open-access article distributed under the terms of the Creative Commons Attribution-NonCommercial 4.0 International License (http://creativecommons.org/licenses/by-nc/4.0/) which permits copy and redistribute the material just in noncommercial usages, provided the original work is properly cited. 


\section{Objectives}

Therefore, this study is intended to contribute to health policy-makers of the country about the optimal decision and policy development in this area, by performing costeffectiveness and cost-utility analysis on anti-rotavirus vaccination for under-5 children.

\section{Patients and Methods}

\subsection{Model Structure}

A decision tree model in Tree-Age pro version 9 was applied to calculate total vaccination cost minus medical cost of diarrhea saved by such an immunization program. In addition we estimated three outcomes of outpatient care, inpatient care, and death. Finally we calculated cost per DALY averted in the 5-year time horizon. Two different vaccination scenarios were determined in the study for two RotaTeq and Rotarix vaccines, which were compared with a scenario without vaccination (Figure 1). For each scenario, four assumptions were considered on the basis of the disease incidence rates and different episodes, for which DALY was separately estimated. The model followed a 2010 Iranian birth cohort of 1,300,000 children.

The treatment costs of rotavirus occurring over 5-year period were estimated. To analyze the costs, the total cost of the vaccination program was estimated in the intervention scenario in the 5-year time horizon. In addition, the direct costs of medical care and treatment as well as the indirect social costs of rotavirus occurring over 5-year period were estimated in non-vaccination scenario on the basis of incidence estimates for each episode scenario, then the medical and treatment cost savings was calculated according to vaccine efficacy. Cost of vaccination program and treatment cost of rotavirus calculated in base year and then adjusted with annual discount rate of 3\% and inflation factor. After all the epidemiological data and the data on cost and efficacy were entered into the model, the costeffectiveness analysis was finally obtained by calculating the cost per DALY averted for every scenario.
All costs were expressed in US dollars. The monetary values in Iranian Rials were converted into United States dollars (USD) based on the average exchange rate in base year 2010 ( 1 USD = 20,000 Rials).

\subsection{Parameter Estimation}

\subsubsection{Epidemiological Data}

Since in general there was no comprehensive statistics on rotavirus diarrhea in Iran, a systematic review and meta-analysis was performed and then the pooled estimates were calculated for proportion of rotavirus gastroenteritis to obtain a relatively comprehensive epidemiological proportion of disease in the country. On the basis of the systematic review overall pooled estimate of infection with rotavirus among cases of gastroenteritis was 0.35 ( $95 \% \mathrm{CI}, 0.28$ - 0.41), pooled estimates for hospitalized children and outpatient visits were 0.39 (95\% CI, 0.30 - 0.48) and 0.31 (95\% CI, 0.23 - 0.38), respectively (18). Diarrhea episodes in Iran were estimated based on four assumptions from the department of IMES database, expert panel and WHO-EMRO estimate. The total number of rotavirus diarrhea cases was calculated by multiplying the diarrhea cases based on four assumptions for the incidence of diarrheal disease in Iran by the percentage of rotavirus infection of about 35\% (Table 1). The software DISMOD was used to estimate other epidemiological parameters. The primary inputs to the software included the overall mortality rate of the country's population on the basis of different age groups separately from the IRMIDHS database, the number of estimated populations in 2010 from population division of the ministry of health and other calculated disease parameters such as disease duration. The resulting outputs of the software were evaluated in terms of plausibility, and the burdens of disease from rotavirus diarrhea were determined for all the assumptions in WHO's DALY computational sheet after modeling the epidemiologic data and fitting the data. Disability weight associated with the disease, time discount rate and different age values were used to calculate DALY (Table 2).

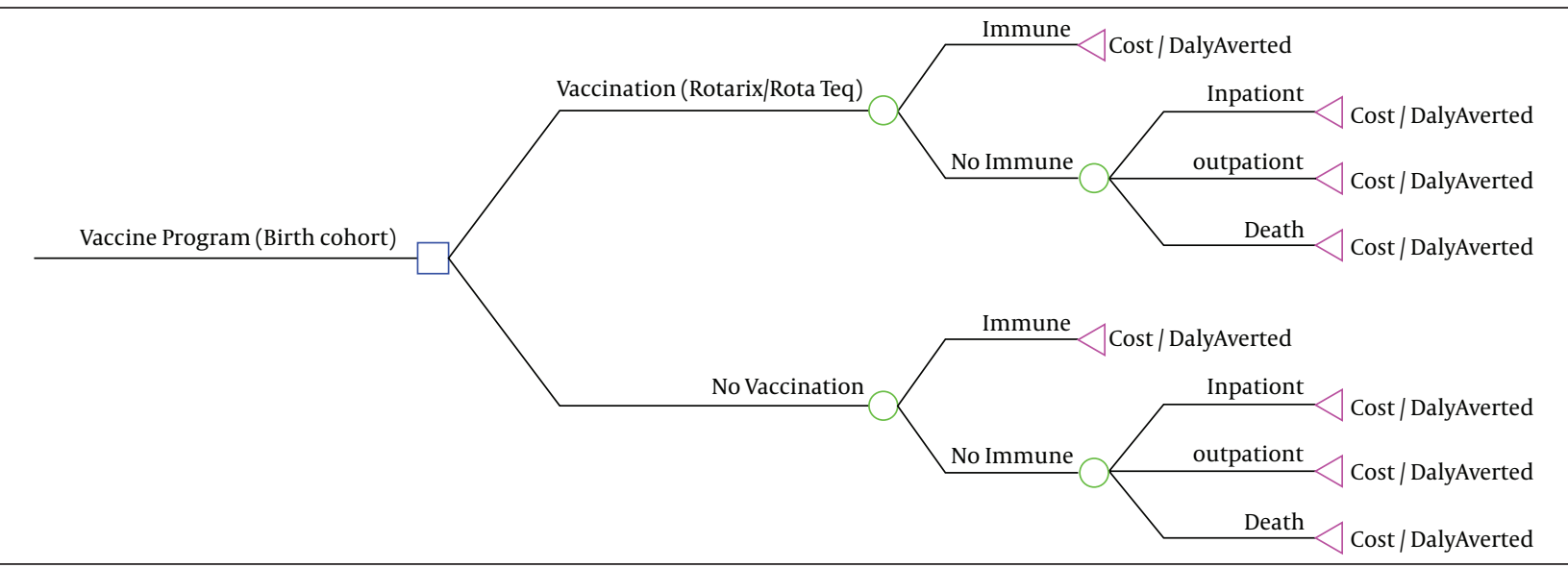

Figure 1. Decision Tree Model Structure for Implementation Anti Rotavirus Vaccination for Under 5 Children in Iran 
Shakerian S et al.

\subsection{Vaccines Efficacy}

The pooled estimates of rotavirus vaccine efficacy in vaccine trials, which were derived during a systematic review, were used for the efficacy and safety of the vaccines. On the basis of systematic review the pooled estimate for vaccine efficacy for rotarix and RotaTeq was 80\% (95\% CI: 0.68 - 0.87) and 70\% (95\%CI: 0.65 - 0.85) respectively against Rotavirus infection. The results were described in detail in efficacy paper (19). Characteristics of studies used to derive the efficacy rate of the RotaTeq and Rotarix vaccines, along with different outcomes, are presented in Table 3.

\subsection{Vaccines Cost}

The costs of providing RotaTeq and Rotarix vaccines were calculated according to the cohort number of live birth per year, the rate of vaccine coverage, the number of doses required for a fully immunized child, the wastage rate, and the price per dose (Table 4 ). Rotavirus vaccine coverage was predicted to be the same as of DPT coverage rates in Iran from CDC database and it was assumed that $98 \%$ of children would be fully immunized. The major costs associated with the implementation of the vaccination program (such as the costs of transportation, cold chain storage, training and stationery, surveillance and maintenance) have been estimated using the models that were based on data from previous vaccinations and the clinical guidelines of the world health organization. Since oral vaccines have been used in the study, the costs of syringes and alcohol were not calculated, and personnel costs were also ignored because of the simultaneous injection of other routine vaccines. We assumed 10 years as useful working life of vehicle and refrigerators along with discount rate to calculate annualized capital system cost. In addition all cost categories in base year were measured for the following 5 years on the basis of predicted value of vaccine price and number of birth cohort and adjusted with inflation and discount factor in Model. Information about costs was obtained from the experts of the ministry of health.

Table 1. Summary of Model Epidemiologic Parameters

\begin{tabular}{|c|c|c|}
\hline Parameter & Value & Source \\
\hline Population of under five children in 2010 & 5948599 & IHM \\
\hline Diarrhea rate Iran 1th scenario & $10.20 \%$ & IHM \\
\hline Diarrhea rate Iran 2th scenario & $17.34 \%$ & IHM \\
\hline Diarrhea rate Iran 3th scenario & $100 \%$ & Expert panel \\
\hline Diarrhea rate Iran 4th scenario & $300 \%$ & EMRO-WHO \\
\hline Proportion of rotavirus infection in acute gastroenteritis & $35 \%(28 \%-41 \%)$ & Calculated -Meta analysis [30] \\
\hline Number of under five mortality due to acute gastroenteritis & 161 & IRMIDHS \\
\hline Number of under five mortality due to rotavirus infection diarrhea & 58 & Literature \\
\hline Under five mortality rate due to rotavirus infection diarrhea per 100000 & 1 & Calculated \\
\hline
\end{tabular}

Table 2. Burden of Disease Parameters

\begin{tabular}{lcccc}
\hline Index & 1th Scenario & 2th Scenario & 3th Scenario & 4th Scenario \\
\hline Total diarrhea rate (episode) in Iran, \% & 10.20 & 17.34 & 100 & 300 \\
Total diarrhea in Iran & 606757 & 1031487 & 5948599 & 17845797 \\
Total number of rotavirus infection diarrhea in Iran & 218433 & 371335 & 2141496 & 6424487 \\
DALY & 21582 & 22494 & 31946 & 56614 \\
\hline
\end{tabular}

Table 3. Summery of Vaccine Efficacies a

\begin{tabular}{lcc}
\hline Vaccine Efficacy & RotaTeq & Rotarix \\
\hline Severe and none severe & 70 & 80 \\
Hospitalization & 90 & 94 \\
Death & 84 & 84 \\
\hline
\end{tabular}

a Data are presented as \%. 
Shakerian $S$ et al.

\begin{tabular}{lccc}
\hline Table 4. Vaccine Cost Parameters & & & \\
\hline Parameter & RotaTeq & Rotarix & Source \\
\hline Number of dose per vial & 1 & 1 & WHO \\
\hline Coverage rate, $\%$ & 98 & 98 & IHM \\
\hline Number of cohort in base year 2010 & $1,300,000$ & $1,300,000$ & IHM \\
\hline Wastage rate factor & 1.05 & 1.05 & WHO \\
\hline Number of doses per fully immunized child & 3 & 2 & WHO \\
\hline The total of number of dose supplied & 4013100 & 2675400 & IHM \\
\hline Price per dose & 7 & 7 & Calculated b \\
\hline Total vaccine supplies cost in base year & 28091700 & 18727800 & IHM \\
\hline Vaccine delivery vehicles & 65000 & 52000 & IHM \\
\hline Cold chain storage & 71624 & 57299 & IHM \\
\hline Training material & 81250 & 81250 & IHM \\
\hline Surveillance & 31250 & 31250 & IHM \\
\hline Redesign of stationery & 93750 & 93750 & IHM \\
\hline Transport maintenance & 48000 & 48000 & IHM \\
\hline Cold chain maintenance & 22997 & 22997 & calculated \\
\hline Total vaccine system cost in 5 Year & 196283401 & 131856059 & \\
\hline
\end{tabular}

a The total of number of dose supplied = birth cohort * coverage rate * number of dose per fully immunized child * wastage rate.

b Total vaccine supplies cost $=$ the total number of dose supplied * price per dose.

\subsection{Health Service Costs}

Direct and indirect treatment costs were calculated for inpatient and outpatient cases in public and private hospitals.The rates of use of inpatient and outpatient services in the public and private units were obtained from the statistics of the ministry of health, which were applied in the calculation. Utilization rates for outpatient services in private units were equal to $67.7 \%$. The rate of use of inpatient units by people was higher in the public sector and was reported at $84 \%$.

Average cost of hospitalization has been calculated based on a sample of 50 patients who were hospitalized for viral diarrhea in Hazrat Rasoul Hospital in Tehran. The inpatient cost included daily room charge, diagnostic tests, medications, inpatient visits and outpatient consultation and out of pocket medical expenditure.

In order to calculate the outpatient services costs we extracted pharmaceutical items, diagnostic tests, and outpatient visits after consultation with expert's panel including health professionals, GPs, pediatric assistants and pediatricians in public and private sector.

Different tariff rates in the public and private sectors have been used to calculate the cost per unit. In addition, the indirect costs of treatment, including the opportunity cost, were calculated using the human capital approach on the basis of data on the duration of diarrheal illnesses as 5 day in hospital and 2 days at home and estimate of daily wages was based on basic wages (USD 5.5/ day) in Iran. After calculation of average cost, total cost for under-5 children was calculated using the incidence rate resulting from modeling in the population of under- 5 children. Given the rate of use of inpatient and outpatient units for different assumptions of incidence, the overall results of values are shown in Table 5.

\subsection{Cost-Effectiveness Analysis}

Analysis of cost effectiveness for rotavirus vaccination was calculated in comparison with the case of no vaccination program with ministry of health prospective. To estimate the incremental cost-effectiveness, the difference between the costs in both scenarios was divided by the difference between the amounts of DALY averted, according to the following formula. In addition, the WHO threshold rate was used to interpret the incremental cost-effectiveness.

ICER = total vaccination cost-Cost saved/DALY without vaccine-DALY with vaccine

\section{Results}

The results of the evaluation of the efficacy of the RotaTeq and Rotarix vaccines, which were obtained from the previous studies, show that the efficacy of RotaTeq vaccine was $0.7,0.9$, and 0.84 for the outcomes of general cases of illness, cases of hospitalization and death cases, respectively, while these rates were obtained at $0.8,0.94$, and 0.84 for the Rotarix vaccine, respectively. The results of the vaccine efficacy are presented in Table 4. Also, results of the systematic review and meta-anal- 
ysis performed in the reference year for the proportion of rotavirus gastroenteritis show that, overall pooled estimate of proportion of rotavirus gastroenteritis in Iran was 0.35 (95 CI: 0.28 - 0.41). Pooled estimate for inpatient and outpatient cases was 0.39 (95 CI: 0.30-0.48) and 0.31 (95 CI: $0.23-0.38$.) respectively. The results of the proportion of rotavirus in Iran in detail are presented in another paper (18).

The vaccine supplies cost of RotaTeq and Rotarix were estimated at USD 192,540,723 and USD 128,360,482 respectively (Table 5). In addition, the total cost of the vaccination program was estimated at USD 196,283,401 and USD 131,856,059 including cold chain storage, transportation, training material, redesign of stationery, surveillance, and maintenance for the RotaTeq and Rotarix vaccines, respectively (Table 4). The incremental system cost of the vaccination program is USD 0.31 for RotaTeq and USD 0.29 for Rotarix. Assuming the episode of disease is $100 \%$, the total number of the patients amounts to $2,141,496$, while the total amount of DALY for the disease is equal to 31,964 . In this case, the total direct and indirect medical costs are equal to USD 48,227,829 (Table 5). Health service cost per children was estimated at USD 63 in general cases, USD 1163 for admissions, and USD 146 for homecare and outpatient treatments. Findings from the economic evaluation showed that assuming the episode of disease is $100 \%$, with a minimum efficacy of $70 \%$ for Rota Teq and $80 \%$ for Rotarix vaccines, can avert 1,499,047 and 1,742,188 cases of disease as well as 22,362 and 25,557 of DALY, respectively (Table 5). Con- sidering the 58 cases of death from rotavirus diarrhea in under-5 children, life-years gained were calculated equal to 3,533 years for both RotaTeq and Rotarix vaccines. For all the outcomes, the social, and medical cost saved have also been estimated at over 33 and 38 million dollars for RotaTeq and Rotarix in the population of under-5 children, respectively. For the amount of the episode above, the costs per DALY averted is equal to 7268 and 3650 , respectively, which represents the highly cost-effectiveness of the vaccination program for Rotarix on the basis of GDP per capita for Iran in 2010 (less than 1 GDP $=4526$ US \$) and cost effective for RotaTeq (less than three times the GDP $=13578$ ). Also, RotaTeq vaccine is highly cost effective assumed that the episode of disease is 300\% (Table 6).

\subsection{Sensitivity Analysis}

We considered uncertainty related to burden of disease parameter (Table 2) and vaccine price per dose. Results of two way sensitivity analysis for cost-effectiveness, which was made for different amounts of vaccine price (USD 10 and USD 15), and the different episode of disease in the four scenarios showed that increasing the vaccine prices up to USD 10, rotavirus vaccination was not cost-effective in low episode of rotavirus (first scenario). When vaccine price rises up to USD 15 RotaTeq vaccine remains cost effective in high episode of disease (100\% and 300\%). Regarding Rotarix in scenario analysis, this vaccine was not cost effective with vaccine price up to USD 15 in lower episode of disease (Table 7).

Table 5. Total Estimated Cost and Health Care Utilization Measures

\begin{tabular}{|c|c|c|c|c|c|c|c|c|c|}
\hline \multirow[t]{2}{*}{ Episode,\% } & \multicolumn{3}{|c|}{ No Vaccine } & \multicolumn{3}{|c|}{ RotaTeq } & \multicolumn{3}{|c|}{ Rotarix } \\
\hline & $\begin{array}{l}\text { Total } \\
\text { Cases }\end{array}$ & $\begin{array}{l}\text { Total } \\
\text { DALY }\end{array}$ & $\begin{array}{l}\text { Social and } \\
\text { Medical } \\
\text { Cost }\end{array}$ & $\begin{array}{c}\text { Total } \\
\text { Cases } \\
\text { Averted }\end{array}$ & $\begin{array}{c}\text { Total } \\
\text { DALY } \\
\text { Averted }\end{array}$ & $\begin{array}{l}\text { Social and } \\
\text { Medical } \\
\text { Cost Saved }\end{array}$ & $\begin{array}{c}\text { Total } \\
\text { Cases } \\
\text { Averted }\end{array}$ & $\begin{array}{c}\text { Total } \\
\text { DALY } \\
\text { Averted }\end{array}$ & $\begin{array}{c}\text { Social and } \\
\text { Medical } \\
\text { Cost Saved }\end{array}$ \\
\hline 10.20 & 218433 & 21582 & 5033395 & 152903 & 15107 & 3523376 & 167419 & 17266 & 4026716 \\
\hline 17.34 & 371335 & 22494 & 8476862 & 259935 & 15746 & 5933803 & 302096 & 17995 & 6781489 \\
\hline 100 & 2141496 & 31946 & 48227830 & 1499047 & 22362 & 33759481 & 1742188 & 25557 & 38582264 \\
\hline 300 & 6424487 & 56614 & 144797645 & 4497141 & 39630 & 101358352 & 5226567 & 45292 & 115838116 \\
\hline
\end{tabular}

Table 6. Cost-Effectiveness Result

\begin{tabular}{|c|c|c|c|c|}
\hline \multirow[t]{2}{*}{ Episode,\% } & \multicolumn{2}{|c|}{ Cost Per DALY Averted } & \multicolumn{2}{|c|}{ Cost Per Case Averted } \\
\hline & RotaTeq & Rotarix & RotaTeq & Rotarix \\
\hline 10.20 & 12760 & 7404 & 1261 & 764 \\
\hline 17.34 & 12089 & 6951 & 732 & 414 \\
\hline 100 & 7268 & 3650 & 108 & 54 \\
\hline 300 & 2395 & 354 & 21 & 3 \\
\hline
\end{tabular}


Shakerian S et al.

\begin{tabular}{lccc}
\hline Table 7. Two Way Sensitivity Analyses & & & \\
\hline Total Rotavirus Episode per 100,000 & Vaccine Price in Base Year ${ }^{\text {a }}$ & ICER RotaTeq & ICER Rotarix \\
\hline $\mathbf{3 6 7 2}$ & 10 & 16186 & 9402 \\
\hline $\mathbf{6 2 4 3}$ & 10 & 15376 & 8868 \\
\hline $\mathbf{3 6 0 0 0}$ & 10 & 9582 & 5000 \\
$\mathbf{1 0 8 0 0 0}$ & 10 & 3701 & 1115 \\
\hline $\mathbf{3 6 7 2}$ & 15 & 23380 & 13599 \\
$\mathbf{6 2 4 3}$ & 15 & 22278 & 12895 \\
\hline $\mathbf{3 6 0 0 0}$ & $15 \mathrm{U}$ & 14443 & 7835 \\
\hline $\mathbf{1 0 8 0 0 0}$ & 15 & 6444 & 2715 \\
\hline a USD. & & &
\end{tabular}

\section{Discussion}

The results of the study showed that in the case of program implementation, the social costs of treatment are reduced by over 70\% in RotaTeq and $80 \%$ in Rotarix. Assuming there are the lowest episode (10 out of every 100 children are affected by diarrhea episodes per year) to the highest number of attacks of diarrhea (three episodes of diarrhea per child per year), the costs prevented by the implementation of program vary from over 4 to 115 million dollars in Rotarix and from about 3 to 101 million dollars in RotaTeq, as district statistics suggest. The number of incidences to the prevented disease has ranged from 167,416 to over 5 million episodes of the illness in Rotarix and 152903 to over 4 million episodes in RotaTeq. In the above rates, cost per DALY averted and cost per case averted (Table 6) represents the effective implementation of the program.

The study's results of the sensitivity analysis (Table 7), also, showed that the incremental cost-effectiveness ratio increases with the vaccine prices, but ultimately, the results still remain cost-effective in higher episode of disease. According to the review, cases of rotavirus gastroenteritis comprise over one third of all cases of acute gastroenteritis admitted to clinics and hospitals in the country. In addition, this level was found to reach approximately $50 \%$ in the hospital studies, which represents high rates of hospitalization due to rotavirus diarrhea and would be an economic burden on the government and the community. Many studies were conducted on economic evaluation of anti-rotavirus vaccines in different parts of the world, most of which have been cost-effective. A sample of the studies conducted in developing countries showed that rotavirus vaccination can annually prevent 228,000 deaths, 13.7 million hospital visits and 8.7 million adjusted life years in terms of disability and can cause approximately USD 188 million savings in treatment costs and 243 million dollars in social costs (20). At the rate of USD 5 per dose, the cost per adjusted year is equal to USD 88, USD 291 and USD 329 in terms the prevented disability for low-income, below average, and above average income countries, respectively (21) with the price of USD 7 per dose of vaccine and the illness episode reported by the ministry of health with a probability of $70 \%$ confidence (the rate has ranged from 114 to 958 for both RotaTeq and Rotarix vaccines in our country). Treatment of rotavirus in Vietnam annually costs about USD 5.3 million for the community. With a cost of approximately USD 7.26 per dose, the vaccination of infants is considered a cost-effective intervention in public health for the health system (22). In the United States, the comprehensive rotavirus vaccination program prevents from 1.08 million cases of acute diarrhea, 34000 hospitalizations, 95,000 cases of emergency admission and 227,000 visits to the doctor in the first 5 years of life (21). In a study to evaluate the economic burden of rotavirus infection, which took into account the burden of disease in under-7 children in some countries such as Belgium, it was expected that rotavirus gastroenteritis annually results in 5,600 hospitalizations ( 679 out of about one hundred thousand children) and 26,800 outpatient visits to pediatricians and GPs. The virus also causes 44600 attacks, whose economic burden is estimated at 7.7 and 12 million USD in direct and indirect costs, respectively (23). In addition, assuming the vaccine price is USD 2 per dose, cost per DALY averted is equal to USD 22 in Uzbekistan, which reaches about USD 117 with the price of USD 10 for each vaccine dose. Given the USD 700 per capita in this country in 2004 , it is considered a costeffective intervention. Given the above prices, the cost for the prevention of death was 734 dollars and 3864 dollars respectively (24). In Iran, the two vaccines are considered as highly cost-effective with regard to the standard criterion of the world health organization for cost-effective interventions (cost per DALY averted is 
Shakerian S et al.

less than the per capita income that is equal to 4,526 dollars). Due to the high rates of vaccine efficacy, especially in cases of hospitalization and severe illness, the decision to implement the program is noteworthy because most of the financial burden in above cases is imposed with the occupancy of hospital beds to the community and government.

One limitation of this study is that many of costs (such as intangible costs and some direct non-medical costs) are not considered due to the problems of estimation in the country. On the other hand, some complications such as rates of emergency admission and nosocomial cases of the disease were not included in the study because of unavailability of reliable epidemiological data in the country. Maybe the preserved costs resulting from the program implementation increase because of all the complications created. It is important to note that rotavirus vaccination is recommended to be applied concurrent with other routine vaccines because, as the reports suggest, it will result in a $30 \%-40 \%$ reduction in the costs of the program implementation $(8,16)$. In terms of the safety of the RotaTeq and Rotarix vaccines, no serious complications were observed, and the main side effects were fever and mild systemic symptoms that can also be seen in other vaccines. Given that in our country, other fever vaccines are commonly prescribed with some routine recommendations at the health centers, the costs of vaccine complications were not calculated in this study with regard to its co-administration with other routine vaccines. As shown in the table, although Rotarix is more cost-effective than RotaTeq, due to the cost-effectiveness of both vaccines, they have different effects on different strains, which should be carefully considered in the decision to select vaccines, considering the strains prevalent in the country. In studies conducted in different regions, it was shown that the RotaTeq vaccine can more affect the strains G1, G2, G3, G4, or P1A while Rotarix vaccine has more impact on the strains G1P (8). According a review of the studies conducted in the country, G4P and G3 are the most commonly reported types of strain (7, 25). However, to determine the strains prevalent in the country, larger studies with greater precision are suggested in order to choose the effective vaccine type.

According the evidences regarding vaccine efficacy and safety for some effect in reducing the risk of severe forms of the disease, rotavirus vaccination based on the threshold set by the world health organization is cost-effective due to the high economic burden of the disease, and, if added to the national vaccination program, will contribute towards optimal allocation of resources.

\section{Acknowledgements}

This study was supported by the Iran national institute of health research (Iran NIHR, grant number: 241/M/471). We express our appreciation to Dr. Arash Rashidian, head of the NIHR.

\section{References}

1. World Health Organization. Initiative for Vaccine Research (IVR) Diarrhoeal Diseases Rotavirus . WHO; 2009. Available from: http:/ www.scribd.com/doc/102430886/WHO-Diarrhoeal-DiseasesUpdated-February-2009.

2. Moradi AV, Tabarrae A, Roshandel GH, Ghaemi EZ, Bazoori M. Rotavirus infection in under 6 years old children with acute diarrhea in Gorgan Iran. Iran J Infect Dis Trop Med. 2010;15(48):55-9.

3. Centers for Disease C, Prevention. Rotavirus surveillance-worldwide, 2001-2008. MMWR Morb Mortal Wkly Rep. 2008;57(46):1255-7.

4. Glass RI, Bresee JS, Turcios R, Fischer TK, Parashar UD, Steele AD Rotavirus vaccines: targeting the developing world. J Infect Dis. 2005;192 Suppl 1:S160-6.

5. Levy K, Hubbard AE, Eisenberg JN. Seasonality of rotavirus disease in the tropics: a systematic review and meta-analysis. Int J Epidemiol. 2009;38(6):1487-96.

6. World Health Organization. New and Under-utilized Vaccines Implementation (NUVI) Rotavirus. 2008. Available from: http://apps. who.int/nuvi/en/.

7. Eesteghamati A, Gouya M, Keshtkar A, Najafi L, Zali MR, Sanaei M, et al. Sentinel hospital-based surveillance of rotavirus diarrhea in iran. J Infect Dis. 2009;200 Suppl 1:S244-7.

8. World Health Organization. [WHO recommends rotavirus vaccine for all children]Editing by Stephanie Nebehay. Geneva: Reuters; 2009. Available from: http://www.reuters.com/article/2009/06/05/usvaccines-rotavirus-idUSTRE5541U620090605.

9. Dennehy PH. Transmission of rotavirus and other enteric pathogens in the home. Pediatr Infect Dis J. 2000;19(10 Suppl):S103-5.

10. World Health Organization. Rotavirus vaccines position paper. 2009. Available from: http://www.who.int/wer/2007/wer8232.pdf.

11. Simpson E, Wittet S, Bonilla J, Gamazina K, Cooley L, Winkler JL. Use of formative research in developing a knowledge translation approach to rotavirus vaccine introduction in developing countries. BMC Public Health. 2007;7:281.

12. Jit M, Edmunds WJ. Evaluating rotavirus vaccination in England and Wales. Part II. The potential cost-effectiveness of vaccination. Vaccine. 2007;25(20):3971-9.

13. Wu CL, Yang YC, Huang LM, Chen KT. Cost-effectiveness of childhood rotavirus vaccination in Taiwan. Vaccine. 2009;27(10):1492-9.

14. World Health Organization-Department of Immunization. Vaccines and Biologicals. CH-1211 Geneva 27 Switzerland: Available from: http://www.who.int/bulletin/volumes/92/5/13-130146/en/.

15. Vesikari T, Giaquinto C, Huppertz HI. Clinical trials of rotavirus vaccines in Europe. Pediatr Infect Dis J. 2006;25(1 Suppl):S42-7.

16. World Healh Organization. Guidelines for estimating economic burden of dirrhoeal disease with focous on assessing rotavirus dirrhoeal. WHO; 2005.

17. Goossens LM, Standaert B, Hartwig N, Hovels AM, Al MJ. The costutility of rotavirus vaccination with Rotarix (RIX4414) in the Netherlands. Vaccine. 2008;26(8):1118-27.

18. Moradi-Lakeh M, Shakerian S, Yaghoubi M, Esteghamati A, Shokraneh F, Baradaran HR, et al. Rotavirus Infection in Children with Acute Gastroenteritis in Iran: A Systematic Review and Meta-analysis. Int J Prev Med. 2014;5(10):1213-23.

19. Shakerian S, Moradi Lakeh M, Esteghamati A, Shokraneh F, Mobinizadeh M, Babashahi S, et al. Vaccine Efficacy against Rotavirus infection in children; systematic review and meta-analysis. J Isfahan Med Sch. 2014;32(303):1622-05.

20. Rheingans RD, Antil L, Dreibelbis R, Podewils LJ, Bresee JS, Parashar UD. Economic costs of rotavirus gastroenteritis and costeffectiveness of vaccination in developing countries.J Infect Dis. 2009;200 Suppl 1:S16-27.

21. Tucker AW, Haddix AC, Bresee JS, Holman RC, Parashar UD, Glass RI. Cost-effectiveness analysis of a rotavirus immunization program for the United States. JAMA. 1998;279(17):1371-6.

22. Fischer TK, Anh DD, Antil L, Cat ND, Kilgore PE, Thiem VD, et al Health care costs of diarrheal disease and estimates of the costeffectiveness of rotavirus vaccination in Vietnam. J Infect Dis. 2005;192(10):1720-6.

23. Bilcke J, Van Damme P, Beutels P. Cost-effectiveness of rotavirus 


\section{Shakerian S et al.}

vaccination: exploring caregiver(s) and "no medical care" disease impact in Belgium. Med Decis Making. 2009;29(1):33-50.

24. Musabaev E, Antil L, Rheingans R, Juraev RI, Glass R, Bresee JS Rotavirus disease in Uzbekistan:Cost-effectiveness of a new vac- cine Vaccine 25. 2007:(1):373-80.

25. Habibi E, Ghorbani S, Jarollahei A, Habibi M, Zali MR. Serotyping of group a rotaviruses in children less than 7 years old in Tehran. The American Journal of Gastroenterology. 2003;98(9):eS73. 